\title{
Atypical Dysphagia in an Anti-Hu Positive Non-Small-Cell Lung Cancer Patient
}

\author{
Inah Kim, M.D. ${ }^{1}$, Jeana Kim, M.D., Ph.D. ${ }^{2}$, Hee Yeon Lee, M.D., Ph.D. ${ }^{3}$, \\ Geun Young Park, M.D., Ph.D. ${ }^{4}$, Sang Ah Jeong, M.D. ${ }^{4}$, Hyemi Hwang, M.D. ${ }^{4}$, \\ Sun Im, M.D., Ph.D. ${ }^{4}$
}

${ }^{1}$ Department of Rehabilitation Medicine, Seoul St. Mary's Hospital, College of Medicine, The Catholic University of Korea, Seoul, 'Department of Hospital Pathology, Bucheon St. Mary's Hospital, College of Medicine, The Catholic University of Korea, Seoul, ${ }^{3}$ Department of Internal Medicine, Yeouido St. Mary's Hospital, College of Medicine, The Catholic University of Korea, Seoul, ${ }^{4}$ Department of Rehabilitation Medicine, Bucheon St. Mary's Hospital, College of Medicine, The Catholic University of Korea, Seoul, Korea

\begin{abstract}
Anti-Hu antibodies usually present in small cell lung cancer are associated with encephalomyelitis, encephalitis, cerebellitis, and neuropathies. However, to our knowledge, non-small-cell lung cancer (NSCLC) related anti-Hu syndrome presenting acute exacerbation of dysphagia has not been reported. Herein, we report a rare case of atypical dysphagia in an anti-Hu positive NSCLC patient resulting in a fatal outcome. A 73-year-old Korean male went through surgical interventions for recurrent tongue cancer and NSCLC. After the surgery, he resumed full oral feeding with a regular diet. However, four weeks later, he developed a sudden difficulty in swallowing. Neck computed tomography angiography, brain magnetic resonance imaging, brain positron emission tomography, computed tomography and electromyography all showed nonspecific findings. A paraneoplastic antibody test was positive for anti-Hu antibodies. His dysphagia worsened and eventually, he expired due to malignant pleural effusion. Our case shows that anti-Hu antibodies can be expressed in NSCLC and when associated with dysphagia, the outcome can be fatal. (JKDS 2022;12:64-69)
\end{abstract}

Keywords: Deglutition disorders, Paraneoplastic syndromes, Carcinoma, Non-small-cell lung

\section{INTRODUCTION}

Anti-Hu antibody is frequently detected in multiple cancers, especially in small cell lung cancer (SCLC) and cause neurological paraneoplastic syndromes, including cerebellar ataxia, limbic encephalitis, Lambert-Eaton syndrome, polyradiculopathy, opsoclonus-myoclonus syndrome, and most commonly, paraneoplastic sen- sory neuropathy ${ }^{1}$. Expression of $\mathrm{Hu}$ antigen in nonsmall-cell lung cancer (NSCLC) is rarely reported and cases associated with dysphagia has not been reported. In this case report, we report a rare case of anti-Hu paraneoplastic syndrome in NSCLC that manifested as rapidly progressive dysphagia. In this study, information pertinent to patient's personal identification were not included. This case report was

Received: June 15 2021, Revised: June 16 2021, Accepted: August 192021

Corresponding author: Sun Im, Department of Rehabilitation Medicine, Bucheon St. Mary's Hospital, College of Medicine, The Catholic University of Korea, 327 Sosa-ro, Wonmi-gu, Bucheon 14647, Korea Tel: +82-32-340-2170, Fax: +82-32-340-2173, E-mail: lafolia@catholic.ac.kr

This is an Open Access article distributed under the terms of the Creative Commons Attribution Non-Commercial License, which permits unrestricted non-commercial use, distribution, and reproduction in any medium, provided the original work is properly cited. 
waived for informed consent due to the retrospective nature of the case.

\section{CASE REPORT}

A 73-year-old male was referred to the department of rehabilitation medicine for the evaluation of dysphagia. He had multiple medical comorbidities including hypertension, dyslipidemia, coronary artery disease and atrial flutter. Two years prior, he had been diagnosed with squamous cell carcinoma of the tongue and had gone through right partial glossectomy, followed by radiation therapy. At that time, he had not experienced any major swallowing difficulties as an aftereffect. Five months prior to our visit, he underwent near total glossectomy due to recurrence of tongue cancer. After the operation the patient resumed regular diet. However, 4 weeks after operation, aspiration symptoms suddenly developed. Aspiration pneumonia occurred and consequently he received percutaneous endoscopic gastrostomy. At the same time, lung cancer in positron emission tomography-computed tomography (PET-CT) was found with biopsy results indicating a double primary cancer of poorly differentiated squamous cell carcinoma in left upper lobe of lung, which is one of the main subtypes of NSCLC.(Fig. 1) In the fiberoptic endoscopic evaluation of swallowing with ice chip and liquid protocol, premature spillage of liquid into the vallecular space was noted. Also, no laryngeal elevation motion was detected. Despite the patient's effort to initiate swallowing, no pharyngeal trigger was observed. Aspiration with weak coughing was shown.(Fig. 2) His videofluoroscopic swallowing study (VFSS) confirmed pharyngoesophageal passage difficulty, very poor pharyngeal stripping waves and impaired pharyngeal wall contraction.(Fig. 3) Laryngeal motion was significantly impaired and overflow aspiration without coughing reflex was observed. These findings did not correlate with the surgical procedures that mainly involved the oral cavities. Head and neck computed tomography angiography, brain magnetic resonance imaging and PET-CT all showed no evidence of occult brain or head and neck lesions that may explain for patient's swallowing difficulty.(Fig. 4, 5) Results of nerve conduction

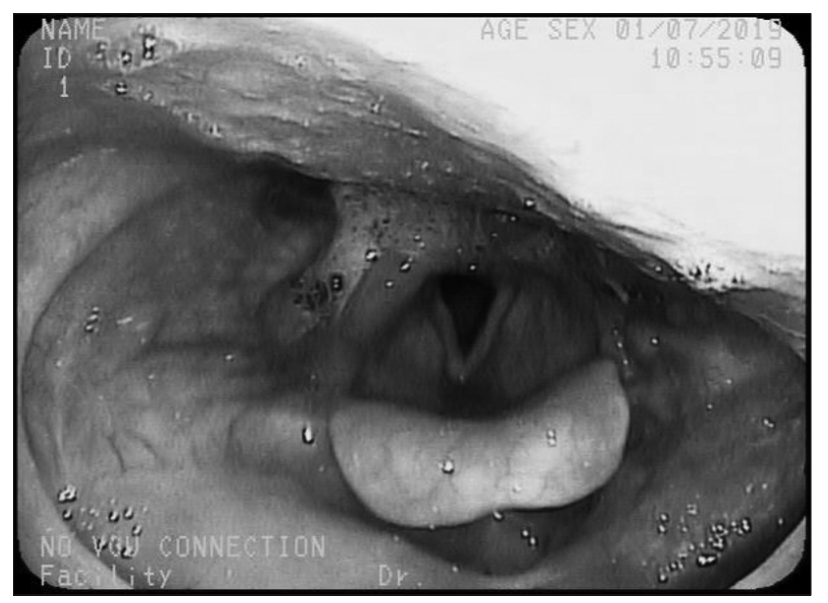

Fig. 2. Fiberoptic endoscopic evaluation of swallowing with ice chip and liquid protocol shows saliva pooling of moderate degree with saliva aspiration past glottis.
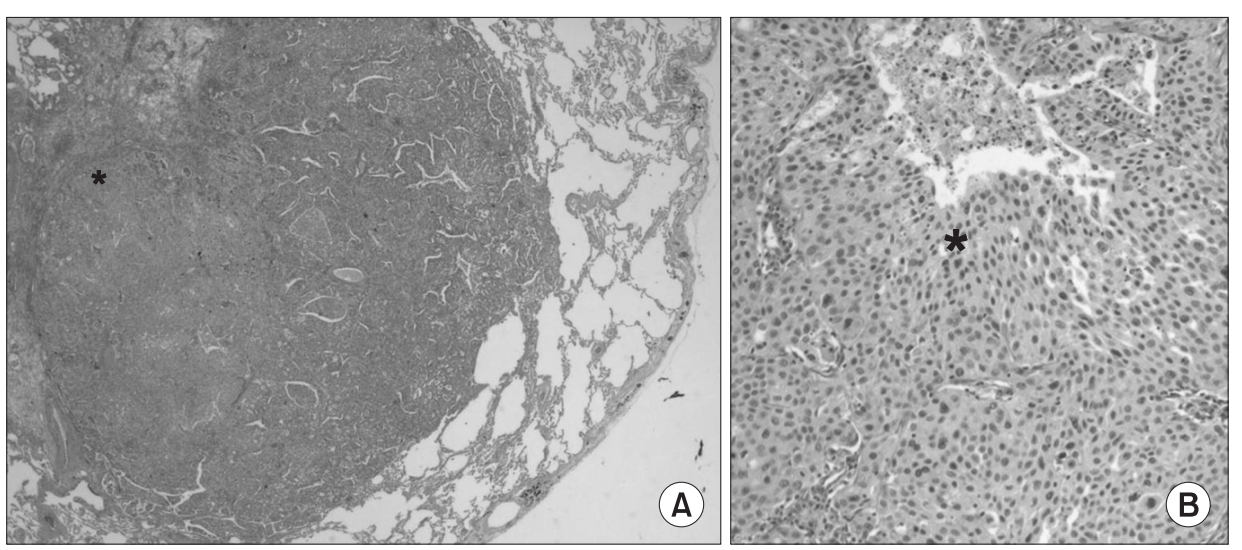

Fig. 1. Hematoxylin and eosin stained lung biopsy shows poorly differentiated squamous cell carcinoma in left upper lobe (asterisk). (A) Magnification $\times 20$. (B) Magnification $\times 100$. 


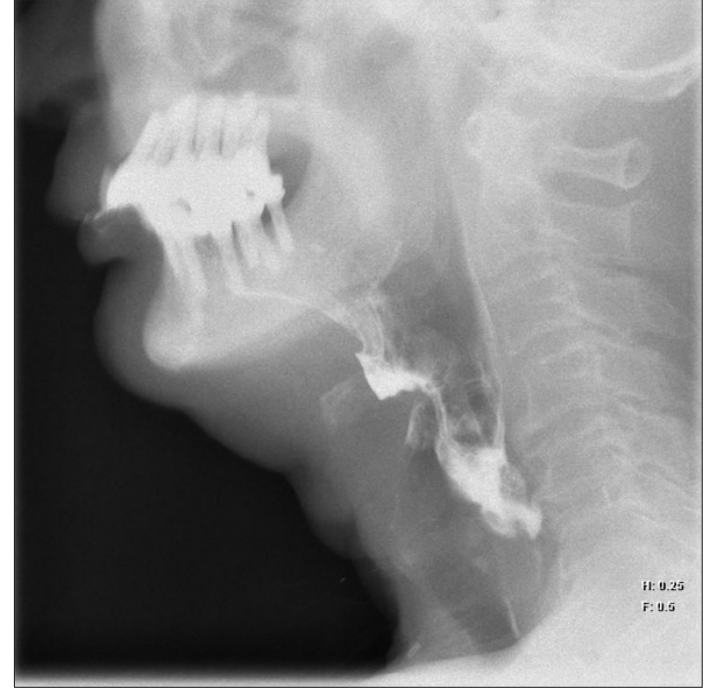

Fig. 3. Videofluoroscopic swallowing study confirmed pharyngoesophageal passage difficulty, very poor pharyngeal stripping waves and impaired pharyngeal wall contraction. Laryngeal motion was significantly impaired and overflow aspiration without coughing reflex was observed.
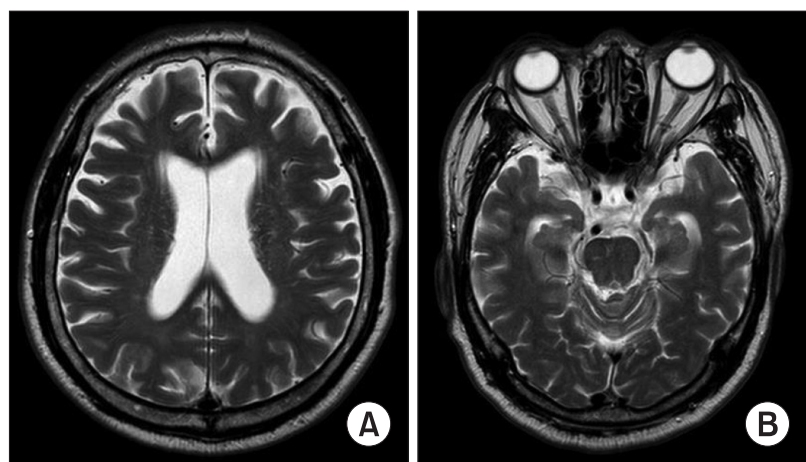

Fig. 4. T2-weighted brain magnetic resonance images in the axial view (A, B) show no acute lesion or neurodegenerative change.

study/needle electromyography showed no evidence of peripheral neuropathy, myopathy or neuromuscular junction disorder. Serum study of paraneoplastic antibody was positive for anti-Hu antibodies with borderline positive titer between 7 and 14. Despite swallowing rehabilitation, the patient showed progressive rapid deterioration of dysphagia in two months in following instrumental swallowing tests. A follow-up titer showed further elevation of anti-Hu antibodies. Soon after, his medical condition rapidly deteriorated with chest computed tomography showing

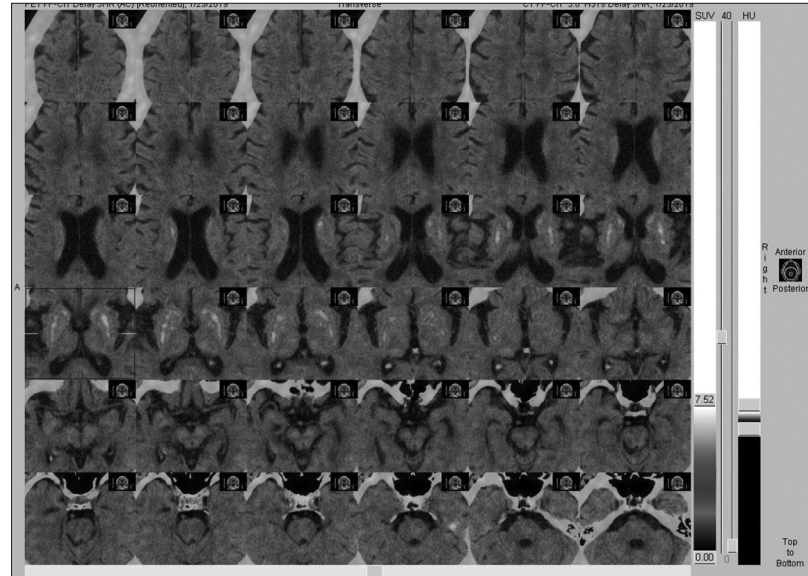

Fig. 5. Brain dopamine transporter (DAT) imaging with positron emission computed tomography shows normal DAT activity in bilateral striata.

increased size of lung mass. The patient expired nine months later due to aspiration pneumonia and complications related to malignant pleural effusion.

\section{DISCUSSION}

We experienced an unusual case of anti-Hu paraneoplastic syndrome related to NSCLC; poorly differentiated squamous cell carcinoma; presenting as atypical dysphagia. The $\mathrm{Hu}$ antigens are a family of nuclear proteins normally expressed in all neurons of the central/peripheral nervous system. The presence of antibodies against $\mathrm{Hu}$ proteins in paraneoplastic syndromes is associated with destruction of parts of the nervous system and with relative control of growth of the underlying tumor ${ }^{2}$. Hu antigen is almost exclusively present in neuronal tissue, but may ectopically be expressed by cancer cells, especially in small-cell lung cancer ${ }^{3}$. Expression of $\mathrm{Hu}$ antigen by the tumor triggers an immune response that is misdirected against similar proteins expressed in brain ${ }^{4}$.

The exact mechanism on how anti-Hu syndrome may lead to swallowing difficulty is unknown. Fujimoto, et al. reported a case of SCLC with high titer of anti-Hu antibodies who developed a tonic left pupil and multiple cranial nerve palsies, including 
palsies of the left fifth through tenth nerves and both twelfth nerves presenting dysphagia ${ }^{5}$. Saiz, et al. reported that among 22 patients with positive anti-Hu antibodies, definite paraneoplastic sensory neuronopathy, clinical presentation compatible with brainstem involvement and tumor diagnosis, a medullary syndrome was seen in 11 patients and 7 of them presented with dysphagia, dysarthria and central hypoventilation ${ }^{6}$. It could be presumed that anti-Hu antibody that attacks proteins similar to $\mathrm{Hu}$ antigen expressed in cranial nerve IX or X, may have caused dysphagia in our patient. This is in accordance to the VFSS features that showed functional impairment of the pharyngeal stages.

Anti-Hu antigen is known to be selectively expressed in SCLC. In SCLC, anti-Hu antibody may rarely result in dysphagia but the prognosis is reported to be favorable ${ }^{1}$. There are two unusual presentations of our case that deserves attention, first; anti-Hu antibodies was related to NSCLC and not to SCLC, second; as dysphagia aggravated, medical condition deteriorated with worsened dyspnea with an increase in titer of anti-Hu antibody. This is in sharp contrast to the protective roles suggested in some studies ${ }^{1} . \mathrm{Ni}$, et al. reported that patients with SCLC and positive anti-Hu antibodies showed tendency to respond better to therapy and live longer than cancer patients who did not possess anti-Hu antibodies, ${ }^{1,113,14}$. Furthermore, SCLC patients with anti-Hu antibody-associated paraneoplastic sensory neuropathy showed extraordinary long-term survival ${ }^{1,11,14}$.

In our case, we concluded that the fast deteriorating nature of dysphagia might have been related to a rare presentation of anti-Hu paraneoplastic syndrome, since other possible causes were excluded. In general, dysphagia associated with cancer often results from direct invasion or metastasis of tumor into the brain. In our case, a structural lesion of the pharyngeal area that may affect airway protection and pharyngeal passage was excluded. Moreover, atypical features of dysphagia in our case could not be explained by post-operative changes alone. The patient had resumed oral diet immediately after the operation. Also, the operation site only involved the oral structure with sparing of the pharyngeal structures. Sudden aggravation 4 weeks after the glossectomy and increased titer of anti-Hu antibody as the deterioration of swallowing progressed were supplying arguments. In fact, Rihani, et al. reported that tongue atrophy occurs in patients who underwent total glossectomy with reconstruction, leading to gradual decrease in swallowing function over time ${ }^{10}$. This is quite different from the dysphagia patterns of our patient. Also, the patient did not have any swallowing problems after the first and the second glossectomy. Therefore, one may cautiously postulate that the rapid aggravation of dysphagia may have been related to paraneoplastic syndrome related to anti-Hu antibody. Until now, only limited studies have shown dysphagia to present in anti-Hu syndrome ${ }^{6,7}$ and this is the first case report to show its relation to NSCLC.

Some limiting factors need to be considered in this study. First, it took several weeks for the final results to be confirmed and unfamiliarity of anti-Hu paraneoplastic syndrome led to a delay in diagnosis. Even though atypical features of dysphagia that did not match with patient's surgical site were detected in swallowing tests, the paraneoplastic antibody studies were performed only after exclusion of other causes, such as stroke or movement disorder. Second, our case did not show any evidence of neuronopathy, one of the typical symptoms of anti-Hu syndrome. However, this does not draw doubts about anti-Hu syndrome in our case, since there are cases when anti-Hu antibody was present and yet no evidence of neuropathy. Some reports have shown that $13 \%$ of these Anti-Hu could present with no neuropathy ${ }^{8}$. According to Kazarian, et al., 16-25\% of SCLC patients without paraneoplastic neurological autoimmune syndromes have detectable titers of anti-Hu antibodies in their serum ${ }^{9}$. In fact, the involvement of the cranial nerve can be confirmed through laryngeal electromyography. Nevertheless, it was not testable in our patient due to severe medical deterioration. However, the involvement of the cranial nerves was 
suspected in this patient by the absence of laryngeal elevation and pharyngeal trigger in fiberoptic endoscopic evaluation of swallowing. Lastly, radiation therapies might have had a delayed effect on dysphagia. However, the first radiotherapy was confined to the tumor bed (60 Gy/30 Fxs), and the second one was confined to the flap area and IV-Vb lymphatics in the left neck (50 Gy/28 Fxs). The radiation dosage and extent may have been too limited to have involved the posterior pharyngeal muscles or hyoid muscles. In fact, it is known that the risk of fibrosis increases with irradiation dose, particularly after total doses exceeding $70 \mathrm{~Gy}^{12}$. Moreover, our patient did not show definite passage difficulty due to stricture in pharyngeal or esophageal phases, which is commonly found in radiotherapy-induced dysphagia. Also, radiation-induced dysphagia manifests with insidious onset with a more chronic clinical course. Despite the accumulation effects of the radiation therapies cannot be completely ruled out, it is unlikely to have been the main cause of swallowing dysfunction considering the acute deteriorating nature as seen in this case. According to our knowledge, our case is one of the few rare cases of anti-Hu positive paraneoplastic syndrome related to NSCLC presenting as atypical dysphagia.

In conclusion, our case suggests that anti-Hu antibody related paraneoplastic syndrome in NSCLC may be related to fatal dysphagia. In contrast to the protective roles seen in SCLC, the contrasting detrimental effects in NSCLC should alert clinicians to its aggressive clinical course. To better understand and manage these medically complex patients, more research is warranted on the exact mechanism of $\mathrm{Hu}$ antigen and its role in NSCLC leading to dysphagia.

\section{CONFLICT OF INTEREST}

No potential conflict of interest relevant to this article was reported.

\section{AUTHOR CONTRIBUTIONS}

Conceptualization: Kim IA, Im S.

Writing-original draft: Kim IA, Kim JA, Im S.

Writing-review \& editing: Kim IA, Im S, Lee HY, Jeong SA, Park GY, Hwang HM.

Approval of final manuscript: All authors.

\section{REFERENCES}

1. Ni J, Weng L, Liu M, Yang H, Wang Y. Small Cell Lung Cancer Accompanied by Tonsillar Metastasis and Anti-Hu Antibody-Associated Paraneoplastic Neuropathy: A Rare Case Report With Long-Term Survival. Medicine (Baltimore). 2015;94:e2291.

2. Hammam T, McFadzean RM, Ironside JW. Anti-hu paraneoplastic syndrome presenting as bilateral sixth cranial nerve palsies. J Neuroophthalmol. 2005;25:101-104.

3. Raskin J, Masrori P, Cant A, Snoeckx A, Hiddinga B, Kohl S, et al. Recurrent dysphasia due to nivolumab-induced encephalopathy with presence of Hu autoantibody. Lung Cancer. 2017;109:74-77.

4. Dalmau J, Furneaux HM, Cordon-Cardo C, Posner JB. The expression of the $\mathrm{Hu}$ (paraneoplastic encephalomyelitis/sensory neuronopathy) antigen in human normal and tumor tissues. Am J Pathol. 1992;141:881-886.

5. Fujimoto S, Kumamoto T, Ito T, Sannomiya K, Inuzuka T, Tsuda T. A clinicopathological study of a patient with anti-Hu-associated paraneoplastic sensory neuronopathy with multiple cranial nerve palsies. Clin Neurol Neurosurg. 2002;104:98-102.

6. Saiz A, Bruna J, Stourac P, Vigliani MC, Giometto B, Grisold W, et al. Anti-Hu-associated brainstem encephalitis. J Neurol Neurosurg Psychiatry. 2009;80:404-407.

7. Yeung JC, Pringle CE, Sekhon HS, Kilty SJ, Macdonald K. Bilateral Vocal Cord Paralysis and Cervicolumbar Radiculopathy as the Presenting Paraneoplastic Manifestations of Small Cell Lung Cancer: A Case Report and Literature Review. Case Rep Otolaryngol. 2016;2016: 2868190.

8. Dalmau J, Graus F, Rosenblum MK, Posner JB. Anti-Hu-associated paraneoplastic encephalomyelitis/sensory neuronopathy. A clinical study of 71 patients. Medicine (Baltimore). 1992;71:59-72.

9. Kazarian M, Laird-Offringa IA. Small-cell lung cancer-associated autoantibodies: potential applications to cancer diagnosis, early detection, and therapy. Mol Cancer. 2011;10:33.

10. Rihani J, Lee T, Ducic Y. Secondary onlay free flap reconstruction of glossectomy defects following initial successful flap restoration. Otolaryngol Head Neck Surg. 2013;149:232-234. 
11. Ni J, Weng L, Liu M, Yang H, Wang Y. Small Cell Lung Cancer Accompanied by Tonsillar Metastasis and Anti$\mathrm{Hu}$ Antibody-Associated Paraneoplastic Neuropathy: A Rare Case Report With Long-Term Survival. Medicine (Baltimore). 2015;94:e2291.

12. Jacques B, Harry K, Henk S, Augustinus H, Joop D, Joos $\mathrm{L}$, et al. Dose and volume effects on fibrosis after breast conservation therapy. Int $\mathrm{J}$ Radiation Oncology biol
Phys. 1994;30:1073-1081.

13. Graus F, Dalmou J, Reñe' R, et al. Anti-Hu antibodies in patients with small-cell lung cancer: association with complete response to therapy and improved survival. J Clin Oncol. 1997;15:2866-2872.

14. Poepel A, Jarius S, Heukamp LC, et al. Neurological course of long-term surviving patients with SCLC and anti-Hu syndrome. J Neurol Sci. 2007;263:145-148. 